\title{
Retrospective Study of Nerve Injury and Pedicle Screw Breach after Pedicle Screw Fixation with Intraoperative Triggered Electromyography Monitoring
}

\author{
Nattawut Niljianskul, M.D. ${ }^{1}$, I-sorn Phoominaonin, M.D. ${ }^{2}$ \\ 'Division of Neurosurgery, Department of Surgery, Faculty of Medicine Vajira Hospital, Navamindradhiraj University, \\ Dusit, Bangkok 10300, Thailand. \\ ${ }^{2}$ Faculty of Science and Health Technology, Navamindradhiraj University, Dusit, Bangkok 10300, Thailand. \\ Received 9 February 2021 • Revised 3 June 2021 • Accepted 3 June $2021 \bullet$ Published online 9 August 2021
}

\begin{abstract}
:
Objective: To evaluate the incidence of nerve injury and pedicle breach after pedicle screw fixation (PSF) with intraoperative triggered electromyography (tEMG) monitoring.

Material and Methods: All patients who underwent PSF with intraoperative tEMG at Vajira Hospital between October 2018 and March 2020 were included. Patients with dysmorphic pedicle features, preoperative infection, or incomplete follow-up data were excluded. PSF was done with intraoperative tEMG. The stimulation threshold was recorded. Stimulation threshold $<7 \mathrm{~mA}$ was not allowed to proceed with the procedure and required reposition of pedicle screw immediately. Post-operative nerve injury was evaluated by physical examination and computer tomography of the spine was done to detect any pedicle breaches. The sensitivity and specificity of intraoperative tEMG to detect pedicle breach were calculated. The risk factors associated with pedicle breach were analyzed.
\end{abstract}

Results: The records of thirty-six patients with 278 pedicle screws were analyzed. No post-operative nerve injuries were found. The incidence of pedicle breach was $2.2 \%$. The sensitivity and specificity were $83.0 \%$ and $91.0 \%$, respectively. The risk factors associated with pedicle breach were degenerative disease and tumor(s) (odds ratio (OR) 3.05, 95\% confidence interval $(\mathrm{Cl}) 1.11-8.41, \mathrm{p}$-value=0.030) and stimulation threshold 7-10 mA (OR 0.02, 95\% Cl 0.00-0.19, p-value< 0.001).

Contact: Nattawut Niljianskul, M.D.

Division of Neurosurgery, Department of Surgery, Faculty of Medicine Vajira Hospital,

Navamindradhiraj University, Dusit, Bangkok 10300, Thailand.

E-mail: nattawut@nmu.ac.th

This is an open access article under the CC BY-NC-ND license

(http://www.jhsmr.org/index.php/jhsmr/about/editorialPolicies\#openAccessPolicy).

J Health Sci Med Res 2022;40(3):251-259 doi: $10.31584 /$ jhsmr.2021832 www.jhsmr.org 
Conclusion: PSF with intraoperative tEMG was safe for neural integrity. Intraoperative tEMG had the ability to detect pedicle breaches with fair sensitivity and high specificity. Patients with degenerative disease, tumors, or stimulation threshold less than $11 \mathrm{~mA}$ had a higher risk of pedicle breach.

Keywords: pedicle breach, pedicle screws, triggered electromyography monitoring

\section{Introduction}

The fixation of pedicle screws is commonly used to treat spinal pathologies such as trauma, degenerative disease, scoliosis, and tumors. The advantages of pedicle screw fixation (PSF) are stability, correct alignment, and improved fusion rates. The accuracy of pedicle screw placement is very important as malposition of the screw and pedicle wall breach could lead to serious complications such as nerve root injury, spinal cord injury, vascular injury, dural laceration, visceral injury, pseudarthrosis, and/or instrument failure. Nerve injury is the most common complication that confronts the spine surgeon. In the literature, other commoncomplication reported is malposition of the pedicle screw, with one study reported an incidence of $42.0 \%{ }^{1}$ and neurological deficit rates between 1.0-11.0\%. ${ }^{2}$ Pedicle breach rates ranged from $10.0-58.0 \%{ }^{3}$ Despite technical advances over the last few decades, PSF is still associated with risk of complications. A number of methods such as fluoroscope and intraoperative navigation-assisted PSF to aid visualization/navigation of the pedicle or laminectomy for pedicle wall palpation to create space and allow for easier pedicle screw placement have become accepted tools to improve the procedure and reduce complications. Electromyography threshold testing is another intervention used to precisely place a PSF.

In 1992 Calencie et al. ${ }^{4}$ introduced intraoperative triggered electromyography (tEMG) by applied electrical stimulus through pedicle screws or instruments and subsequent measurement of muscle action potentials from myotomes innervated by nearby nerve roots, then reported the result of an evoked electromyography (EMG) stimulation threshold. An irritated or damaged nerve root would cause a decrease in this threshold. Maguire et al. ${ }^{5}$ concluded that an EMG threshold of $\leq 6 \mathrm{~mA}$ was the optimal sensitivity cutoff for detecting a malpositioned screw. Initially tEMG was applied to assure correct placement of a lumbosacral PSF. Subsequently this technique has been applied to thoracic PSFs, cervical lateral mass screws and iliosacral screw fixation.

The purpose of this research was to study the efficacy of intraoperative tEMG during PSF to protect neural integrity and detect potential pedicle wall breaches during free-hand PSF technique.

\section{Material and Methods}

After approval by the Institution's Ethics Committee (027/63), patients aged 16-85 years who underwent PSF with intraoperative tEMG monitoring at Vajira Hospital between October 2018 and March 2020 were included. The indications for PSF were trauma, degenerative disease, scoliosis, and tumor(s). Patients with dysmorphic pedicle features such as congenital scoliosis, preoperative infection, or incomplete follow-up data were excluded.

Preoperative $\mathrm{X}$-rays and magnetic resonance imaging spines were obtained for all patients. General anesthesia was done without a neuromuscular blocker or agents that affect neuromuscular monitoring. Intraoperative neuromuscular monitoring was done with an NVM5 ${ }^{\circledR}$ machine (NUVASIVE, San Diego, USA) to evaluate somatosensoryevoked potentials, transcranial motor-evoked potentials, 
and electromyography. A train of four twitches was done at the common peroneal nerve. $A$ response rate $\geq 75.0 \%$ was required before tEMG recording was begun.

All screws were placed by a single experienced spinal neurosurgeon using a free-hand technique. The entry point depended on the spinal level as follows:

Cervical spine: lateral mass screws inserted at $1 \mathrm{~mm}$ below and $1 \mathrm{~mm}$ medial to the midpoint of the lateral mass directed to the upper outer quadrant of the lateral mass.

Thoracic spine: entry point made at $3 \mathrm{~mm}$ caudal to the junction of transverse process and superior articular facet.

Lumbosacral spine: entry point made at the junction of the pars interarticularis, mamillary process, midpoint of transverse process, on lateral border of facet joint.

After an entry point was identified, the pedicle probe was advanced into the vertebral bodies through the pedicles. A pedicle-sounding device was used to palpate the 5 bony borders (medial, lateral, proximal, distal and floor) of the pedicle hole. A tapper was used to tap pedicle holes and pedicle-sounding device was palpated again. Stimuli were applied by attaching a dynamic stimulation clip to the instruments including a pedicle probe, tapper, and screw driver (Figure 1). The tEMG threshold was obtained in rectangular, monophasic pulse waveform, a constant current of pulse width 200 microseconds $\pm 2.0 \%$ at a rate of 5 hertz $(\mathrm{Hz})$. Stimulation occurred at $5 \mathrm{~Hz}$ at varying current intensities following a patented rapid hunting algorithm. Information from the tEMG was displayed both in waveform and pictorial color-coded mode on the system's monitor. The color coding for the response threshold current values was divided into 3 groups: green for responses greater than $10 \mathrm{~mA}$, yellow for responses between 7 to $10 \mathrm{~mA}$, and red for responses less than $7 \mathrm{~mA}$ (Figure 2).

Pedicle screws activating the red threshold $(<7 \mathrm{~mA})$ were immediately repositioned and retested. Finally, pedicle screw placement was confirmed intraoperatively by direct visualization, tactile palpation of the pedicle walls and neural structures, and fluoroscopy after completing the operation. Stimulation thresholds through the instruments with the dynamic stimulation clip were recorded.
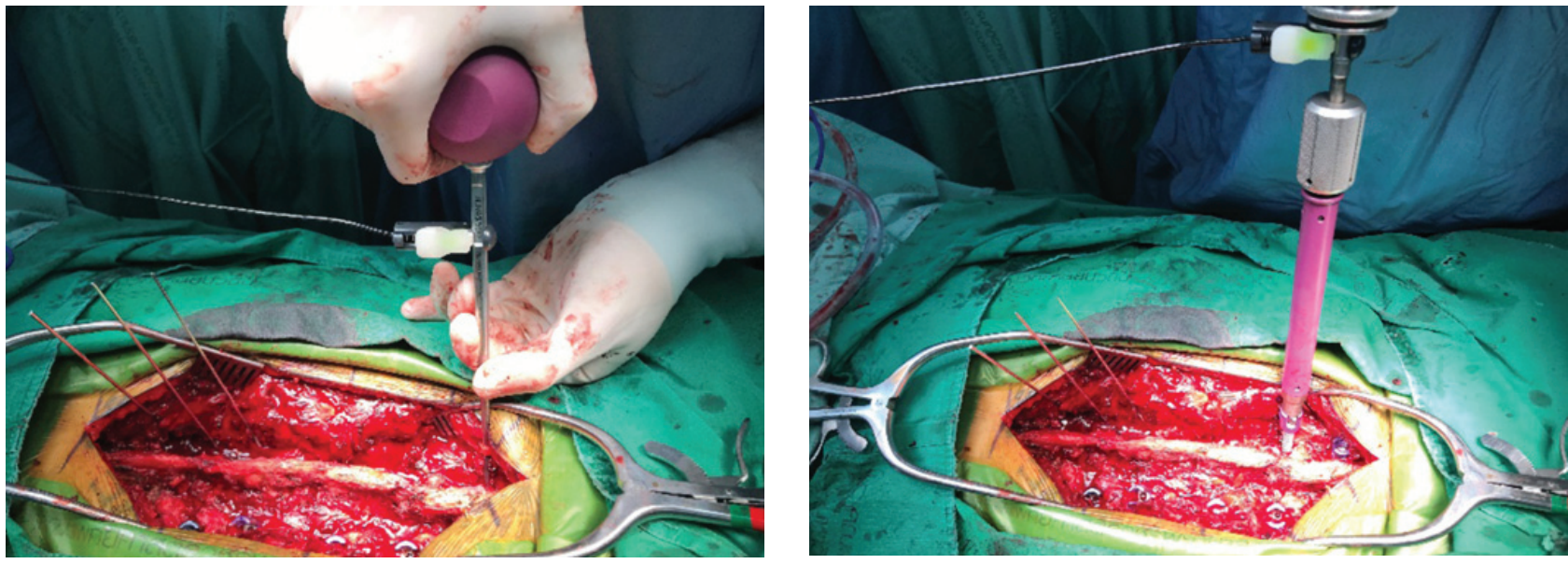

Figure 1 Intraoperative triggered electromyography (tEMG)

The stimulation triggered through a pedicle probe and pedicle screwdriver by dynamic stimulation clip.

(A) Pedicle probe with dynamic stimulation clip. (B) Pedicle screwdriver with dynamic stimulation clip. 


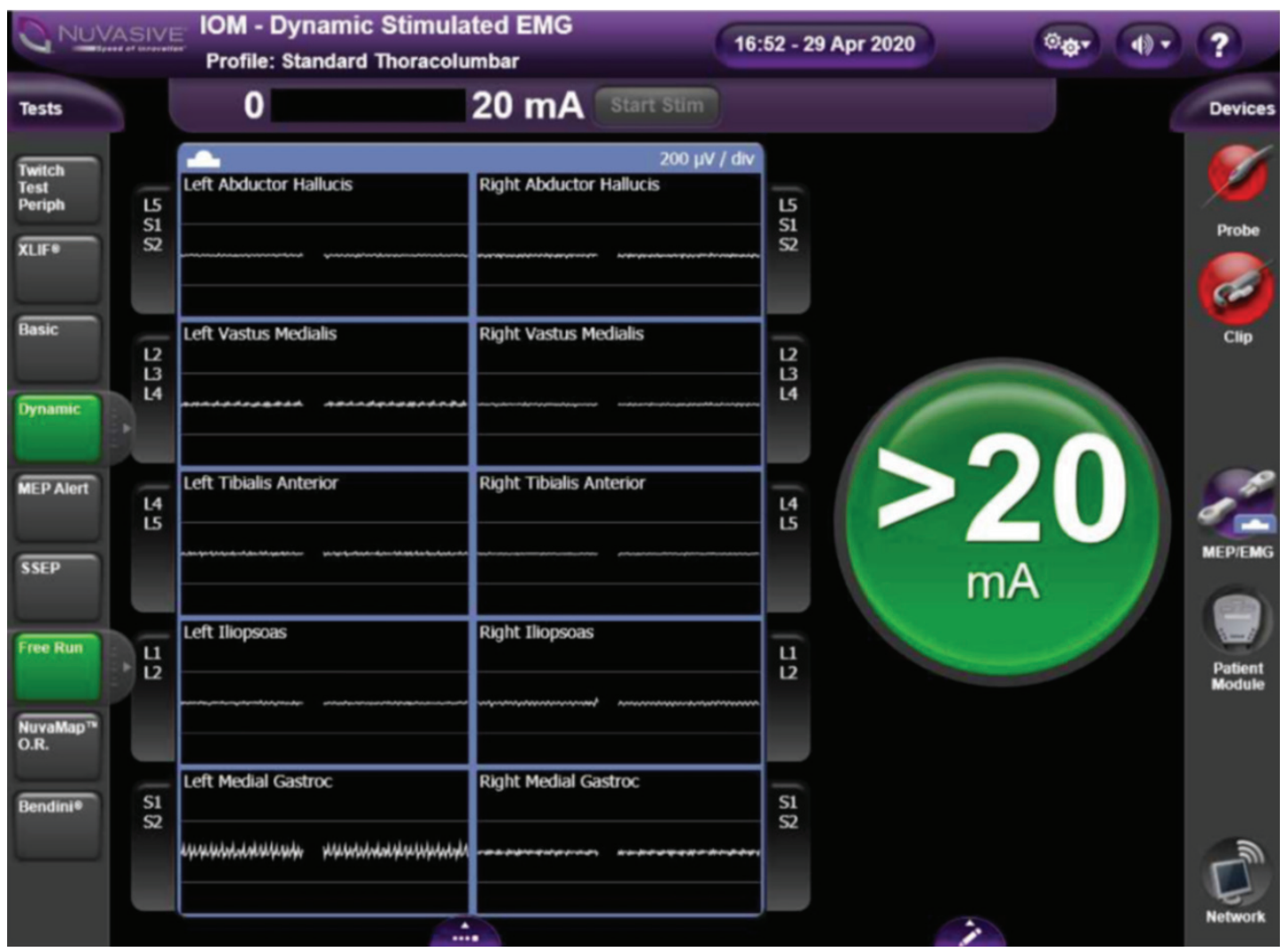

Figure 2 Intraoperative triggered electromyography (tEMG) monitor shows color coding for the response threshold current values

Confounding factors for tEMG monitoring were controlled. Attempts were made to maintain a dry test surgical field, to prevent soft tissue contact with all instruments attached to the dynamic stimulation clip, keep temperature between $36-38^{\circ} \mathrm{C}$ and mean arterial pressure between 65-85 $\mathrm{mmHg}$.

All patients were evaluated by a physical examination and a computerized tomography (CT) scan at the level of the instrumented spine. The CT scans were reviewed by two neurosurgeons. The location of the pedicle screws was assessed and rated according to the criteria of Gertzbein and Robbins ${ }^{6}$, who suggested that a margin of $4 \mathrm{~mm}$. adjacent to the pedicle could be violated safely without impinging on the spinal cord or nerve root.

Statistical analysis was performed with the Statistical Package for Social Science program version 22 for Windows (SPSS Inc., Chicago, IL, USA). Univariate analysis and multivariate analysis were performed with logistic regression. A p-value of $<0.050$ was considered statistically significant. Sensitivity and specificity were calculated. 


\section{Results}

Patients demographics and pedicle screw characteristic

Forty patients who met the inclusion criteria were identified, of whom four were then excluded (1 dysmorphic pedicle feature, 2 preoperative infections, 1 incomplete postoperative imaging). Thirty-six patients with 278 pedicle screws were analyzed. Patient demographic data are shown in Table 1. The mean age of the patients was $51.6 \pm 17.2$ years, and mean body mass index (BMI) was $24.1 \pm 4.3 \mathrm{~kg} /$ $\mathrm{m}^{2}$. The most common indications for PSF were trauma (36.1\%) and degenerative disease (33.3\%). 38.9\% had no underlying disease. The characteristics of the pedicle screws are shown in Table 2. The most common screw location was the lumbosacral spine (51.8\%). The stimulation threshold by using a dynamic stimulation clip attached to the instruments above $10 \mathrm{~mA}$ was achieved in 249 pedicle screws $(89.6 \%)$.

Table 1 Patient demographic data

\begin{tabular}{ll}
\hline Characteristic & $\mathrm{N}=36$ \\
\hline Age (years) & $51.6 \pm 17.2$ \\
Sex & \\
$\quad$ Male & $17(42.2 \%)$ \\
Female & $19(52.8 \%)$ \\
Body mass index $\left(\mathrm{kg} / \mathrm{m}^{2}\right)$ & $24.1 \pm 4.3$ \\
Diagnosis & \\
Trauma & $13(36.1 \%)$ \\
Scoliosis & $5(13.9 \%)$ \\
Degenerative disease & $12(33.3 \%)$ \\
Tumors & $6(16.7 \%)$ \\
Underlying disease & \\
None & $17(38.9 \%)$ \\
Osteoporosis & $7(19.4 \%)$ \\
Type Il diabetes & $5(13.9 \%)$ \\
Other & $10(27.8 \%)$ \\
Operation & \\
Pedicle screw fixation & $26(72.2 \%)$ \\
Lateral mass screw fixation & $10(27.8 \%)$ \\
Operation time, minutes & $300(120-650)$ \\
Blood loss (ml) & $350(10-2,500)$ \\
Blood transfusion (units) & $0(0-2)$ \\
\hline
\end{tabular}

Table 2 Characteristics of pedicle screw

\begin{tabular}{ll}
\hline Characteristic & $\mathbf{N}=\mathbf{2 7 8}(\%)$ \\
\hline Diagnosis & $112(40.3)$ \\
Trauma & $42(15.1)$ \\
Scoliosis & $66(23.7)$ \\
Degenerative disease & $58(20.9)$ \\
Tumors & \\
Underlying disease & $114(41.0)$ \\
None & $52(18.7)$ \\
Osteoporosis & $42(15.1)$ \\
Type II diabetes & $70(25.2)$ \\
Others & \\
Operation type & $220(79.1)$ \\
Pedicle screw fixation & $58(20.9)$ \\
Lateral mass screw fixation & \\
Vertebral level & $58(20.9)$ \\
Cervical spine & $76(27.3)$ \\
Thoracic spine & $144(51.8)$ \\
Lumbosacral spine & \\
Stimulation threshold (mA) & $29(10.4)$ \\
$7-10$ & $249(89.6)$ \\
$>10$ &
\end{tabular}

$\mathrm{mA}=$ milliampere(s)

\section{Surgical complications and risk factors}

No post-operative neurological deficits, infections, or hematomas were found in this cohort. One patient $(2.8 \%)$ had post-operative cerebrospinal fluid leakage after revision surgery. The reoperation rate in this series was zero. Six pedicle breaches $(2.2 \%)$ occurred (cervical spine 2 , thoracic spine 2, lumbar spine 1, and sacrum 1). All were lateral breaches with a breach distance $<4 \mathrm{~mm}$. The potential risk factors were age, sex, BMI, diagnosis, underlying disease, operation type, vertebral level, and stimulation threshold (Table 3). Diagnosis (OR 3.05 [95\% Cl 1.11-8.41]; p-value $=0.030$ ) and stimulation threshold (OR $0.02[95 \% \mathrm{Cl} 0.00-$ 0.19]; $p$-value $<0.001$ ) were associated with pedicle breach. The multivariate analysis is shown in Table 4, in which both diagnosis and stimulation threshold maintained the association with pedicle breach ( $p$-value $<0.050$ for both factors). Degenerative disease and tumors had higher risks 
of pedicle breach, $4.6 \%$ and $5.2 \%$, respectively. The lower stimulation threshold (7-10 $\mathrm{mA}$ ) had a higher risk of pedicle breach $(20.8 \%$ in $7-10 \mathrm{~mA}$ vs. $0.4 \%$ in $>11 \mathrm{~mA})$

\section{Stimulation threshold predicting pedicle breach}

Five breached pedicles were in the lower stimulation threshold group (7-10 mA). One breached pedicle was in the higher stimulation threshold group $(>10 \mathrm{~mA})$. At the stimulation threshold $\geq 7 \mathrm{~mA}$, the sensitivity and specificity of intraoperative tEMG to detect a potential pedicle breach were $83.0 \%$ and $91.0 \%$, respectively. The positive predictive value (PPV) was $17.0 \%$ and the negative predictive value (NPV) was 99.0\% (Table 5). PPV refers to the likelihood of pedicle breach after PSF when the stimulation threshold was $<7 \mathrm{~mA}$. NPV refers to the likelihood of the pedicle wall remaining intact after PSF when the stimulation threshold was $\geq 7 \mathrm{~mA}$.

Table 3 Univariate analysis of potential factors associated with pedicle breach

\begin{tabular}{|c|c|c|c|}
\hline Factor & Number $(\%)$ & OR $(95 \% \mathrm{Cl})$ & p-value \\
\hline Age (years) & & $2.44(0.48-12.36)$ & 0.280 \\
\hline$<60$ & 3/196 (1.5) & & \\
\hline$\geq 60$ & 3/82 (3.7) & & \\
\hline Sex & & $1.40(0.25-7.78)$ & 0.700 \\
\hline Male & $2 / 114(1.8)$ & & \\
\hline Female & 4/164 (2.4) & & \\
\hline Body mass index & & $0.31(0.09-1.12)$ & 0.070 \\
\hline$<18.5$ & $2 / 42(4.8)$ & & \\
\hline $18.5-24.9$ & $4 / 148(2.7)$ & & \\
\hline$\geq 25$ & $0 / 88(0.0)$ & & \\
\hline Diagnosis & & $3.05(1.11-8.41)$ & 0.030 \\
\hline Trauma & $0 / 112(0.0)$ & & \\
\hline Scoliosis & $0 / 42(0.0)$ & & \\
\hline Degenerative disease & $3 / 66(4.6)$ & & \\
\hline Tumors & $3 / 58(5.2)$ & & \\
\hline Underlying disease & & $1.07(0.65-1.74)$ & 0.800 \\
\hline None & $2 / 144(1.4)$ & & \\
\hline Osteoporosis & $0 / 52(0.0)$ & & \\
\hline Type II diabetes & $3 / 42(7.1)$ & & \\
\hline Others & $1 / 70(1.4)$ & & \\
\hline Operation type & & $1.39(0.59-3.29)$ & 0.460 \\
\hline Pedicle screw fixation & $4 / 220(1.8)$ & & \\
\hline Lateral mass screw fixation & $2 / 58(3.5)$ & & \\
\hline Vertebral level & & $0.63(0.24-1.65)$ & 0.340 \\
\hline Cervical spine & $2 / 58(3.5)$ & & \\
\hline Thoracic spine & $2 / 76(2.6)$ & & \\
\hline Lumbar spine & $1 / 126(0.8)$ & & \\
\hline Sacral spine & $1 / 18(5.6)$ & & \\
\hline Stimulation threshold (mA) & & $0.02(0.00-0.19)$ & $<0.001^{*}$ \\
\hline $7-10$ & $5 / 24(20.8)$ & & \\
\hline$>10$ & $1 / 248(0.4)$ & & \\
\hline
\end{tabular}

p-value calculated by logistic regression.

${ }^{*} \mathrm{p}-$ value $=0.000471$

$\mathrm{OR}=$ Odds ratio, $\mathrm{Cl}=$ confidence interval 
Table 4 Multivariate analysis of potential factors associated with pedicle breach

\begin{tabular}{lccc}
\hline Factors & Number (\%) & OR (95\%Cl) & p-value \\
\hline Diagnosis & & $2.85(1.03-7.92)$ & $<0.045$ \\
$\quad$ Trauma & $0 / 112(0.0)$ & & \\
Scoliosis & $0 / 42(0.0)$ & & \\
$\quad$ Degenerative disease & $3 / 66(4.6)$ & & \\
$\quad$ Tumors & $3 / 58(5.2)$ & & \\
Stimulation threshold (mA) & & & \\
$7-10$ & $5 / 24(20.8)$ & & \\
$>10$ & $1 / 248(0.4)$ & & \\
\hline
\end{tabular}

p-value calculated by logistic regression.

$\mathrm{mA}=$ milliampere(s), OR=odds ratio, $\mathrm{Cl}=$ confidence interval

Table 5 Sensitivity and specificity analysis

\begin{tabular}{llll}
\hline Threshold $(\mathbf{m A})$ & Pedicle breach & Intact pedicle & Total PDS \\
\hline $7-10$ & 5 & 24 & 29 \\
$>10$ & 1 & 248 & 249 \\
Sensitivity $83.0 \%$ & Specificity $91.0 \%$ & PPV $17.0 \%^{*}$ & NPV $99.0 \%^{* *}$ \\
\hline
\end{tabular}

${ }^{*} \mathrm{PPV}=$ positive predictive value, ${ }^{* *} \mathrm{NPV}=$ negative predictive value, $\mathrm{PDS}=$ pedicle screw

\section{Discussion}

One study reported the neurological deficit rate after PSF was $1.0-11.0 \% .^{2}$ Other studies have reported that using intraoperative tEMG, the neurological deficit was reduced to $0.0-2.2 \% .^{7-10}$ In this study, using intraoperative tEMG resulted in zero neurological deficits after PSF (0.0\%), further supporting the hypothesis that PSF with intraoperative tEMG is safe for neural integrity.

A meta-analysis of intraoperative tEMG used for detecting misplaced pedicle screws showed fair sensitivity of $78.0 \%$ with high specificity of $94.0 \%$ from different instruments. ${ }^{11}$ With fair sensitivity, intraoperative tEMG can be a helpful monitoring device for detecting potential neural structure injury due to pedicle breach. The sensitivity and specificity for detecting a potential pedicle breach with intraoperative tEMG in this study were $83.0 \%$ and $91.0 \%$, respectively, comparable to previous report. ${ }^{11}$ Many authors recommended stimulation threshold $\geq 6 \mathrm{~mA}$ based on animal study. ${ }^{4,7}$ Prospective clinical series indicated pedicle screw was totally within pedicle with stimulation threshold $\geq 6 \mathrm{~mA}$ and lower stimulation threshold indicated potential pedicle breach. ${ }^{5,12,13}$ From this study, the negative predictive value was $99.0 \%$, suggesting that a pedicle breach is highly unlikely when the stimulation threshold is $\geq 7 \mathrm{~mA}$. The high specificity of our study indicated that a lower stimulation threshold $(<7 \mathrm{~mA})$ has a high chance of pedicle breach and requires screw repositioning. It is possible that the low positive predictive value $(17.0 \%)$ in this study reflected the low incidence of pedicle breach (2.2\%).

In exploratory analysis, this study found that diagnosis and stimulation threshold were associated with pedicle breach (Table 4). A high pedicle breach rate was found in patients with degenerative disease or tumors. This may have resulted from more deformed anatomical 
landmarks of the spine in patients with degenerative disease or tumor than trauma or scoliosis, so the entry point for PSF was difficult to identify. Cordemans et al. used an intraoperative CT technique to detect pedicle breaches which they found in $11.7 \%$ of their cases (81/695). ${ }^{14}$ They also found that non-degenerative diseases including trauma, scoliosis, tumors and infection had higher pedicle breach rates. Both the Cordemanns et al. study and this study found that disease was a risk factor for pedicle breach. However, non-degenerative disease had a higher risk in the Cordemanns study while degenerative disease and tumors had higher risks in this study. This may result from the different techniques and/or surgeon experience. Seventy percent of their patients had degenerative disease versus $33.3 \%$ in this study. A high pedicle breach rate was also found in patients with relatively low stimulation thresholds $(7-10 \mathrm{~mA})$. This could be because electrical stimuli can pass through a pedicle screw, and muscles that are innervated by nearby nerve roots could produce action potentials with low thresholds. A nerve root irritated or damaged by a pedicle screw breach would cause a decrease in this threshold. For these reasons, we suggest using a stimulation threshold $\geq 11 \mathrm{~mA}$ as an optimal cut off to ensure a safe PSF as this level indicates an intact pedicle wall. Belmont et al. recommended the acceptable limits for preventing neurological deficit were $2 \mathrm{~mm}$ for medial wall breach and $6 \mathrm{~mm}$ for lateral wall breach. ${ }^{3}$ All breached pedicles (6 pedicles) in this study were lateral breach and all met the Belmont suggestion (distance $<4 \mathrm{~mm}$ ). Further supported by the evidence that none of the patients with breach in this study had post-operative neurological deficit. However, one silent breached pedicle was detected from the post-operative CT scan without a previously alarm from the intraoperative tEMG, which was a false negative from the intraoperative tEMG. This may have been caused by attached the dynamic stimulation clip too quickly to the instruments. The tEMG monitor failure to detect the change of stimulation threshold. To avoid this problem, we suggest gradually applying all instruments during the stimulation test and carefully examining the pedicle holes prior to pedicle screw placement.

The author recommends that PSF with intraoperative tEMG should be used in conjunction with intraoperative tactile palpation of the pedicle walls and neural structures after decompression and radiography, and using a stimulation threshold $\geq 11 \mathrm{~mA}$ to ensure optimal and safe PSF. The authors accept a lower stimulation threshold but not lower than $7 \mathrm{~mA}$ at the sacral area or osteoporosis patients because the quality of bone in these patients or areas permits more stimuli than normal bone.

This study had several limitations in this study. First, this was a retrospective study with a limited number of patients, thus we could not control for missing data and it was underpowered. Authors did not compare variations in the size of the pedicle screws or other material that may have affected the current threshold resulting in inaccurate predictions of the potential of pedicle breach, possibly also impacting the sensitivity and specificity of the predicting tool. Furthermore, we did not attempt to evaluate underlying diseases which may have affected the bone quality or chronically compressed nerve roots, factors which could affect the current threshold and should be studied in the future.

\section{Conclusion}

PSF with intraoperative tEMG was safe for neural integrity. Intraoperative tEMG had the ability to detect pedicle breaches with fair sensitivity and high specificity. Patients with degenerative disease, tumors, or stimulation threshold less than $11 \mathrm{~mA}$ had higher risk of pedicle breach.

\section{Conflict of interest}

None 


\section{References}

1. Hicks JM, Singla A, Shen FH, Arlet V. Complications of pedicle screw fixation in scoliosis surgery: a systematic review. Spine 2010;35:E465-70.

2. Esses SI, Sachs BL, Dreyzin V. Complications associated with the technique of pedicle screw fixation. A selected survey of ABS members. Spine 1993;18:2231-9.

3. Belmont PJ Jr, Klemme WR, Robinson M, Polly DW Jr. Accuracy of thoracic pedicle screws in patients with and without corona plane spinal deformities. Spine 2002;27:1558-66.

4. Calancie B, Lebwohl N, Madsen P, Klose KJ. Intraoperative evoked EMG monitoring in an animal model. A new technique for evaluating pedicle screw placement. Spine 1992;17:1229_ 35

5. Maguire J, Wallace S, Madiga R, Leppanen R, Draper V. Evaluation of intrapedicular screw position using intraoperative evoked electromyography. Spine 1995;20:1068-74.

6. Gertzbein SD, Robbins SE. Accuracy of pedicular screw placement in vivo. Spine 1990;15:11-4.

7. Lenke LG, Padberg AM, Russo MH, Bridwell KH, Gelb DE. Triggered electromyographic threshold for accuracy of pedicle screw placement. An animal model and clinical correlation. Spine 1995;20:1585-91.

8. Wang MY, Pineiro G, Mummaneni PV. Stimulus-evoked electromyography testing of percutaneous pedicle screws for the detection of pedicle breaches: a clinical study of 409 screws in 93 patients. J Neurosurg Spine 2010;13:600-5.
9. Holdefer RN, Heffez DS, Cohen BA. Utility of evoked EMG monitoring to improve bone screw placements in the cervical spine. J Spinal Disord Tech 2013;26:E163-9.

10. Moed BR, Ahmad BK, Craig JG, Jacobson GP, Anders MJ. Intraoperative monitoring with stimulus-evoked electromyography during placement of iliosacral screws. An initial clinical study. J Bone Joint Surg Am 1998;80:537-46.

11. Mikula AL, Williams SK, Anderson PA. The use of intraoperative triggered electromyography to detect misplaced pedicle screws: a systematic review and meta-analysis. J Neurosurg Spine 2016;24:624-38.

12. Rodriguez-Olaverri JC, Zimick NC, Merola A, De Blas G, Burgos J, Piza-Vallespir G, et al. Using triggered electromyographic threshold in the intercostal muscles to evaluate the accuracy of upper thoracic pedicle screw placement (T3-T6). Spine 2008;33:E194-7.

13. Raynor BL, Lenke LG, Kim Y, Hanson DS, Wilson-Holden TJ, Bridwell $\mathrm{KH}$, et al. Can triggered electromyograph thresholds predict safe thoracic pedicle screw placement? Spine 2002; 27:2030-5

14. Cordemans V, Kaminski L, Banse X, Francq BG, Cartiaux O. Accuracy of a new intraoperative cone beam CT imaging technique (Artis zeego II) compared to postoperative CT scan for assessment of pedicle screws placement and breaches detection. Eur Spine J 2017;26:2906-16. 